\title{
Belnap-Dunn semantics for natural implicative expansions of Kleene's strong three-valued matrix II. Only one designated value
}

\author{
Gemma Robles*, Francisco Salto* and José M. Méndez** \\ *Dpto. de Psicología, Sociología y Filosofía, Universidad de León. \\ Campus de Vegazana, s/n, 24071, León, Spain. \\ gemma.robles@unileon.es; http://grobv.unileon.es. \\ ORCID: 0000-0001-6495-0388 \\ francisco.salto@unileon.es \\ ORCID: 0000-0001-6316-1774 \\ **Universidad de Salamanca. \\ Edificio FES, Campus Unamuno, 37007, Salamanca, Spain. \\ sefus@usal.es; http://sites.google.com/site/sefusmendez \\ ORCID: 0000-0002-9560-3327
}

\begin{abstract}
This paper is a sequel to "Belnap-Dunn semantics for natural implicative expansions of Kleene's strong three-valued matrix with two designated values", where a "bivalent" Belnap-Dunn semantics is provided for all the expansions referred to in its title. The aim of the present paper is to carry out a parallel investigation for all natural implicative expansions of Kleene's strong 3-valued matrix now with only one designated value.

Keywords: Belnap-Dunn type bivalent semantics; Kleene's strong 3valued matrix; natural conditionals; 3 -valued logics; paracomplete logics.
\end{abstract}

\section{Introduction}

This paper is a sequel to [16]. Let us provisionally refer by MK3 ${ }^{1}$ (resp., MK3 ${ }^{2}$ ) to the set of all natural implicative expansions of Kleene's strong 3-valued matrix, MK3, with only one designated value (resp., with two designated values). Furthermore, let us provisionally use LK $3^{1}$ (resp., LK $3^{2}$ ) to refer to the set of all logical systems determined by the matrices $\mathrm{MK}^{1}{ }^{1}$ (resp., MK3 ${ }^{2}$ ). There are exactly 24 systems in $\mathrm{LK}^{2}$ and 6 in $\mathrm{LK} 3^{1}$.

Following [7], in [16] it is shown how to use a Belnap-Dunn type bivalent semantics (BD-semantics) to define all the logical systems in LK3 ${ }^{2}$, which are proved (strongly) sound and complete w.r.t. each respective BD-semantics. The 
aim of the present paper is to develop a parallel investigation in the case of the systems in $\mathrm{LK}^{1}$.

The systems in $\mathrm{LK} 3^{1}$ differ considerably from those in $\mathrm{LK} 3^{2}$. On the one hand, all logics in $\mathrm{LK}^{2}$ are paraconsistent in the sense that the "E contradictione quodlibet" rule, ECQ, i.e.,

$$
A \wedge \neg A \Rightarrow B
$$

fails in each one of them. Conversely, ECQ holds in each logic in LK3 ${ }^{1}$. On the other hand, the 6 logics in LK $3^{1}$ are paracomplete in the sense that prime, consistent theories built upon any of the 6 logics can be incomplete, that is, they can lack both some formula and its negation, since the "Principle of Excluded Middle", PEM, i.e.,

$$
A \vee \neg A
$$

is not a theorem of any of the logics in LK3 ${ }^{1}$. Finally, the 24 systems of LK3 ${ }^{2}$ can be axiomatized by using no more rules of inference than Adjunction and Modus Ponens. However, the 6 systems in $\mathrm{LK} 3^{1}$ can be axiomatized in a general and unified way only if weak rules of inference are used, which complicates the formulation and proof of the Extension and Primeness Lemmas (cf. Section 6, below), and, consequently, the completeness proofs. The facts just pointed out suggested to develop the systems in $\mathrm{LK}^{1}$ and $\mathrm{LK}^{2}$ in two different papers. We have leaned upon some of the definitions and facts recorded in [16], but the present paper is self-contained and can be read independently of its antecedent.

In [16], the fundamental notions "Belnap-Dunn type 'bivalent' semantics", "Kleene's strong 3-valued matrix", and "natural conditional" are explained with some detail. Let us briefly recall them.

Belnap-Dunn type 'bivalent' semantics (BD-semantics) is characterized by the possibility of assigning $T$ (truth), $F$ (falsity), both $T$ and $F$ or neither $T$ nor $F$ to the formulas of a given logical language, or, indeed, to the propositions of a language in general (cf. [5], [6], [8], [9] and references therein). Belnap and Dunn's approach has been generalized in the notion of a bilattice, which has been fruitfully applied in artificial intelligence (cf. [2], [3] and references therein).

On the other hand, Kleene's strong 3-valued matrix MK3 was defined in [12] in the context of the treatment of partial recursive functions. The matrix MK3 (our label) can be defined as shown in Definition 3.1 below. We use the digits 0 (falsity), 2 (truth) and 1, which can represent neither truth not falsity if only 2 is taken as designated value, or both truth and falsity, if 1 is also a designated value, in addition to 2 . Conjunction, disjunction and negation are the only propositional connectives in MK3.

In the third place, a conditional is "natural" if it fulfills the three following conditions. (1) It coincides with the classical conditional when restricted to the classical values $T$ and F; (2) it satisfies the Modus Ponens; and (3) it is assigned a designated value whenever the value assigned to its antecedent is less 
than or equal to the value assigned to its consequent ${ }^{1}$. The notion of a "natural conditional" is understood as defined in [20], but it has to be noted that stricter notions can be found in the literature (cf. [4]).

Given that $\wedge, \vee$ and $\neg$ are the only propositional connectives in MK3, the question of how to expand it with a conditional connective arises. There are, of course, several possibilities, some of which are briefly commented upon in [16]. Here, however, as in the precedent paper, we shall limit ourselves to expansions of MK3 by means of a natural conditional, this notion being understood as explained above. In particular, in what follows, we shall consider all natural implicative expansions of MK3 when 2 is the only designated value.

These "natural implicative expansions" of MK3 will be interpreted by means of a BD-semantics. Actually, by a variant of it in which formulas can be assigned $T, F$ or neither $T$ nor $F$. (In [16], we used another possible variant of BD semantics in which formulas can be assigned $T, F$ or both $T$ and $F$. We have dubbed this second variant "overdetermined (BD-semantics)", the first one being named "underdetermined (BD-semantics)"; cf. [15], [17].)

The aim of this paper is then to define all the logics in $\mathrm{LK}^{1}$ by using a u-determined BD-semantics.

As in the precedent paper [16], we have treated all logics determined by the implicative expansions of MK3 from a general and unified point of view. But, as remarked above, the present paper is self-contained and can be read independently of its predecessor.

Before proceeding to explain the structure of the paper, let us remark the following fact. In [14], BD-semantics for truth functional expansions of Anderson and Belnap's First Degree Entailment Logic, FDE, Priest's LP and Kleene's K3 is systematically discussed. Then, in [19], natural deduction systems for the first-order Belnap-Dunn logics are systematically explored by essentially using Baaz' delta operator. However, in the present paper, we concentrate on defining Hilbert-style systems for the natural implicative expansions of MK3 considered ${ }^{2}$.

The structure of this paper is as follows. In $\S 2$, we define some preliminary notions as used in the paper. In $\S 3$, Kleene's strong 3-valued matrix is recalled. In $\S 4$, the notion of a "natural conditional" (according to [20]) is introduced and all natural implicative expansions of MK3 (with only one designated value) are defined. In $\S 5$, three basic logics are presented and some of their properties are proved. All the logics characterized by the natural implicative expansions of MK3 defined in $\S 4$ (let us name them Lt $i$ logics) are axiomatized as extensions of the basic logics. Some of these logics have not been investigated antecedently in the literature (cf. $\S 10$ ). In $\S 6$, the extension and primeness lemmas are proved. We follow the strategy in [18] (cf. also [7]). As remarked above, it is to be noted that these lemmas (and their proof) are here considerably more involved than those in [16], the reason why being that the Lt $i$-logics are axiomatized with weak rules of inference. In $\S 7$, we define the Lt $i$-logics and prove some of their properties, while in $\S 8$, an underdetermined BD-semantics is defined for each

\footnotetext{
${ }^{1}$ Tomova adds a fourth condition: for any $a, b \in \mathcal{V}, a \rightarrow b \in \mathcal{V}$, in other cases.

${ }^{2}$ The facts just commented were brought to our attention by a referee of the JANCL to whom we sincerely thank.
} 
one of them and the soundness theorems are proved. In $\S 9$, the completeness theorems are proved by means of a canonical model construction. Finally, in $\S 10$, the paper is ended with some concluding remarks on some of the results obtained.

\section{Preliminary notions}

In this section, we record some preliminary notions as used in the present paper (of course, there are alternative definitions of these notions).

Definition 2.1 (Language) The propositional language consists of a denumerable set of propositional variables $p_{0}, p_{1}, \ldots, p_{n}, .$. , and some or all of the following connectives $\rightarrow$ (conditional), $\wedge$ (conjunction), $\vee$ (disjunction), $\neg$ (negation). The biconditional $(\leftrightarrow)$ and the set of wffs are defined in the customary way. A, B etc. are metalinguistic variables.

Definition 2.2 (Logics) A logic $L$ is a structure $\left(\mathcal{L}, \vdash_{L}\right)$ where $\mathcal{L}$ is a propositional language and $\vdash_{L}$ is a (proof-theoretical) consequence relation defined on $\mathcal{L}$ by a set of axioms and a set of rules of inference. The notions of 'proof' and 'theorem' are understood as it is customary in Hilbert-style axiomatic systems $\left(\Gamma \vdash_{L} A\right.$ means that $A$ is derivable from the set of $w f f s$ in $L$; and $\vdash_{L} A$ means that $A$ is a theorem of $L$ ).

Definition 2.3 (Extensions and expansions of a logic $\mathbf{L}$ ) Let $\mathcal{L}$ and $\mathcal{L}^{\prime}$ be two propositional languages. $\mathcal{L}^{\prime}$ is a strengthening of $\mathcal{L}$ if the set of wffs of $\mathcal{L}$ is a proper subset of the set of wffs of $\mathcal{L}^{\prime}$. Next, let $L$ and $L^{\prime}$ be two logics built upon the propositional languages $\mathcal{L}$ and $\mathcal{L}^{\prime}$, respectively. Moreover, suppose that all axioms of $L$ are theorems of $L^{\prime}$ and all primitive rules of inference of $L$ are provable in $L^{\prime}$. Then, $L^{\prime}$ is an extension of $L$ if $\mathcal{L}$ and $\mathcal{L}^{\prime}$ are the same propositional language; and $L^{\prime}$ is an expansion of $L$ if $\mathcal{L}^{\prime}$ is an strengthening of $\mathcal{L}$. An extension $L^{\prime}$ of $L$ is a proper extension if $L$ is not an extension of $L^{\prime}$.

Definition 2.4 (Logical matrix) $A$ (logical) matrix is a structure $(\mathcal{V}, D, \mathrm{~F})$ where (1) $\mathcal{V}$ is a (ordered) set of (truth) values; (2) $D$ is a non-empty proper subset of $\mathcal{V}$ (the set of designated values); and (3) $\mathrm{F}$ is the set of $n$-ary functions on $\mathcal{V}$ such that for each $n$-ary connective $c$ (of the propositional language in question), there is a function $f_{c} \in \mathrm{F}$ such that $\mathcal{V}^{n} \rightarrow \mathcal{V}$.

Definition 2.5 (M-interpretation, M-consequence, M-validity) Let $M$ be a matrix for (a propositional language) $\mathcal{L}$. An $M$-interpretation $I$ is a function from the set of all wffs to $\mathcal{V}$ according to the functions in $\mathrm{F}$. Then, for any set of wffs $\Gamma$ and wff $A, \Gamma \vDash_{M} A$ ( $A$ is a consequence of $\Gamma$ according to $M$ ) iff $I(A) \in D$ whenever $I(\Gamma) \in D$ for all $M$-interpretations $I^{3}$.

\footnotetext{
${ }^{3}$ So $I(\Gamma) \in D$ iff $I(B) \in D$ for each $B \in \Gamma$. In particular, $\vDash_{\mathrm{M}} A$ ( $A$ is M-valid; $A$ is valid in the matrix M) iff $I(A) \in D$ for all M-interpretations $I$. By $\vDash_{\mathrm{M}}$ we shall refer to the relation defined in $\mathrm{M}$.
} 
Definition 2.6 (Logics determined by matrices) Let $\mathcal{L}$ be a propositional language, $M$ a matrix for $\mathcal{L}$ and $\vdash_{L}$ a (proof theoretical) consequence relation defined on $\mathcal{L}$. Then, the logic L (cf. Definition 2.2) is determined by $M$ iff for every set of wffs $\Gamma$ and wff $A, \Gamma \vdash_{L} A$ iff $\Gamma \vDash_{M} A$. In particular, the logic $L$ (considered as the set of its theorems) is determined by $M$ iff for every wff $A$, $\vdash_{L} A$ iff $\vDash_{M} A$ (cf. Definition 2.5).

\section{Kleene's strong 3-valued matrix with only one designated value}

Kleene's strong 3-valued matrix with only one designated element, MK3, can be defined as shown in Definition 3.1.

Definition 3.1 (MK3) The propositional language consists of the connectives $\wedge, \vee$ and $\neg$. Kleene's strong 3-valued matrix with only one designated element, $M K 3$, is the structure $(\mathcal{V}, D, F)$ where (1) $\mathcal{V}=\{0,1,2\}$ and it is ordered as shown in the following lattice

$$
\left.\right|_{0} ^{2}
$$

(2) $D=\{2\}$; (3) $\mathrm{F}=\left\{f_{\wedge}, f_{\vee}, f_{\neg}\right\}$ where $f_{\wedge}$ and $f_{\vee}$ are defined as glb (or lattice meet) and lub (or lattice joint), respectively, and $f_{\neg}$ is an involution with $f_{\neg}(2)=0, f_{\neg}(0)=2$ and $f_{\neg}(1)=1$. We display the tables for $\wedge, \vee$ and $\neg$ :

\begin{tabular}{|c|c|c|c|c|c|c|c|c|c|}
\hline$\wedge$ & 0 & 1 & 2 & $V$ & 0 & 1 & 2 & $\neg$ & 0 \\
\hline 0 & 0 & 0 & 0 & 0 & 0 & 1 & 2 & 0 & 2 \\
\hline 1 & 0 & 1 & 1 & 1 & 1 & 1 & 2 & 1 & 1 \\
\hline 2 & 0 & 1 & 2 & 2 & 2 & 2 & 2 & 2 & 0 \\
\hline
\end{tabular}

The notions of an MK3-interpretation, MK3-consequence and MK3-validity are defined according to the general Definition 2.5.

Recall that, as pointed out in the introduction of this paper, we now use 2 for truth and 1 for neither, whereas 0 represents falsity. (We use the symbols 0,1 and 2 -instead of $0,1 / 2$ and 1 because they are convenient for using the tester in [10], in case the reader needs one.)

Now, let K3 be the logic determined by MK3. A BD-semantics for K3 is defined similarly as in [16], Section 3. Notice, however, that $K$ is the set $\{\{T\},\{F\}, \emptyset\}$ here, instead of being $\{\{T\},\{F\},\{T, F\}\}$, as in [16].

It is well-known that the set of valid wffs is empty if we define the conditional in MK3 similarly as it is defined with disjunction and negation in classical logic. 
In this paper, we consider alternative ways of expanding MK3 with a conditional connective. In particular, all natural implicative expansions of MK3 will be investigated. The notion of a natural conditional is defined in the following section.

\section{Natural implicative expansions of MK3}

Following Tomova [20], we define "natural conditionals" as follow.

Definition 4.1 (Natural conditionals) Let $\mathcal{L}$ be a propositional language with $\rightarrow$ among its connectives and $M$ be a matrix for $\mathcal{L}$ where the values $x$ and $y$ represent the maximum and the infimum in $\mathcal{V}$ in the classical sense. Then, an

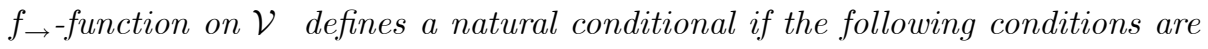
satisfied:

1. $f_{\rightarrow}$ coincides with (the $f_{\rightarrow}$-function for) the classical conditional when restricted to the subset $\{x, y\}$ of $\mathcal{V}$.

2. $f \rightarrow$ satisfies Modus Ponens, that is, for any $a, b \in \mathcal{V}$, if $a \rightarrow b \in D$ and $a \in D$, then $b \in D$.

3. For any $a, b \in \mathcal{V}, a \rightarrow b \in D$ if $a \leq b$.

We have:

Proposition 4.2 (Natural conditionals in 3-valued matrices) Let $\mathcal{L}$ be a propositional language and $M$ a 3-valued matrix for $\mathcal{L}$ where $\mathcal{V}$ and $D$ are defined exactly as in MK3. Now, consider the $6 f_{\rightarrow}$-functions defined in the following general table:

\begin{tabular}{ll|lll}
\multirow{2}{*}{$T I$} & $\rightarrow$ & 0 & 1 & 2 \\
\cline { 2 - 5 } & 0 & 2 & 2 & 2 \\
& 1 & $a$ & 2 & 2 \\
& 2 & 0 & $b$ & 2
\end{tabular}

where $a \in\{0,1,2\}$ and $b \in\{0,1\}$. The set of functions contained in TI is the set of all natural conditionals definable in $M$.

Proof. It is obvious.

Next, the notion of a natural implicative 3 -valued matrix is defined and a proposition collecting the 6 natural implicative expansions of MK3 is proved.

Definition 4.3 (Natural implicative 3-valued matrices) Let $\mathcal{L}$ be a propositional language with the connective $\rightarrow$. And let $M$ be a 3-valued matrix where $\mathcal{V}$ and $D$ are defined as in Definition 3.1. Moreover, let $f \rightarrow$ be one of the functions (defining one of the conditionals) in TI (in Proposition 4.2). Then, it is said that $M$ is a natural implicative 3-valued matrix ${ }^{4}$.

\footnotetext{
${ }^{4}$ Notice that we are supposing that $\mathcal{V}$ is ordered as stated in Definition 3.1.
} 
Proposition 4.4 (Natural implicative expansions of MK3) There are exactly 6 natural implicative expansions of $M K 3, M t 1, M t 2, \ldots, M t 6$, which are defined as follows. Each Mti $(1 \leq i \leq 6)$ is the structure $(\mathcal{V}, D, F)$ where $\mathcal{V}, D, f_{\wedge}, f_{\vee}$ and $f_{\neg}$ are defined exactly as in MK3 (cf. Definition 3.1), whereas $f_{\rightarrow}$ is defined according to the table ti. Tables $t 1, t 2, \ldots, t 6$ are displayed below; the notions of an Mti-interpretation, etc. are defined according to the general Definition 2.5.

Proof. Immediate by Proposition 4.2 and Definition 4.3.

List of the 6 tables:

t1

\begin{tabular}{l|lll}
$\rightarrow$ & 0 & 1 & 2 \\
\hline 0 & 2 & 2 & 2 \\
1 & 2 & 2 & 2 \\
2 & 0 & 1 & 2
\end{tabular}

t4

\begin{tabular}{l|lll}
$\rightarrow$ & 0 & 1 & 2 \\
\hline 0 & 2 & 2 & 2 \\
1 & 1 & 2 & 2 \\
2 & 0 & 1 & 2
\end{tabular}

t2

\begin{tabular}{l|lll}
$\rightarrow$ & 0 & 1 & 2 \\
\hline 0 & 2 & 2 & 2 \\
1 & 2 & 2 & 2 \\
2 & 0 & 0 & 2
\end{tabular}

$\mathrm{t} 3$

\begin{tabular}{l|lll}
$\rightarrow$ & 0 & 1 & 2 \\
\hline 0 & 2 & 2 & 2 \\
1 & 0 & 2 & 2 \\
2 & 0 & 0 & 2
\end{tabular}

$\mathrm{t} 5$

\begin{tabular}{l|lll}
$\rightarrow$ & 0 & 1 & 2 \\
\hline 0 & 2 & 2 & 2 \\
1 & 0 & 2 & 2 \\
2 & 0 & 1 & 2
\end{tabular}

t6

\begin{tabular}{l|lll}
$\rightarrow$ & 0 & 1 & 2 \\
\hline 0 & 2 & 2 & 2 \\
1 & 1 & 2 & 2 \\
2 & 0 & 0 & 2
\end{tabular}

The aim of this paper is to provide an underdetermined BD-semantics for the logic Lt $i$ characterized by each matrix $\operatorname{Mt} i(1 \leq i \leq 6)$

\section{$5 \quad$ The basic logics $\mathbf{b}^{3}, \mathbf{b}_{1}^{3}, \mathbf{b}_{2}^{3}$}

In this section, the basic logics $\mathrm{b}^{3}, \mathrm{~b}_{1}^{3}, \mathrm{~b}_{2}^{3}$ are defined and some of their properties are proved. The logic $\mathrm{b}^{3}$ is axiomatized as follows (the label is intended to abbreviate "basic logic contained in all natural implicative expansions of K3").

Definition 5.1 (The basic logic $\mathbf{b}^{3}$ ) The logic $b^{3}$ is axiomatized with the following axioms and rules of inference ${ }^{5}$ :

Axioms:

A1. $(A \wedge B) \rightarrow A /(A \wedge B) \rightarrow B$

A2. $[(A \rightarrow B) \wedge(A \rightarrow C)] \rightarrow[A \rightarrow(B \wedge C)]$

A3. $A \rightarrow(A \vee B) / B \rightarrow(A \vee B)$

A4. $[(A \rightarrow C) \wedge(B \rightarrow C)] \rightarrow[(A \vee B) \rightarrow C]$

A5. $[A \wedge(B \vee C)] \rightarrow[(A \wedge B) \vee(A \wedge C)]$

A6. $\neg(A \vee B) \leftrightarrow(\neg A \wedge \neg B)$

$A \% . \neg(A \wedge B) \leftrightarrow(\neg A \vee \neg B)$

A8. $A \leftrightarrow \neg \neg A$

\footnotetext{
${ }^{5}$ Notice that " \&" is used as a metalinguistic "and".
} 
Rules of inference:

$$
\begin{gathered}
\text { Adjunction (Adj): } A \& B \Rightarrow A \wedge B \\
\text { Modus Ponens (MP): } A \rightarrow B \& A \Rightarrow B \\
\text { Disjunctive Modus Ponens (dMP): } C \vee(A \rightarrow B) \& C \vee A \Rightarrow C \vee B \\
\text { Disjunctive Transitivity (dTrans): } D \vee(A \rightarrow B) \& D \vee(B \rightarrow C) \Rightarrow D \vee(A \rightarrow C) \\
\text { Disjunctive ECQ (dECQ): } C \vee(A \wedge \neg A) \Rightarrow C \vee B
\end{gathered}
$$

Proposition 5.2 (Some theorems and rules provable in $\mathbf{b}^{3}$ ) The following rules and theorems are provable in $b^{3}$ :

$$
\begin{aligned}
& \text { Transitivity (Trans). } A \rightarrow B \& B \rightarrow C \Rightarrow A \rightarrow C \\
& E C Q . \wedge \neg A \Rightarrow B \\
& \text { Elimination of Disjunction }(E \vee) . A \rightarrow C \& B \rightarrow D \Rightarrow(A \vee B) \rightarrow(C \vee D) \\
& \text { Introduction of Conjunction }(I \wedge) . A \rightarrow C \& B \rightarrow D \Rightarrow(A \wedge B) \rightarrow(C \wedge D) \\
& \text { Summation (Sum). } A \rightarrow B \Rightarrow(C \vee A) \rightarrow(C \vee B) \\
& \text { t1. } A \rightarrow A \\
& \text { t2. } A \leftrightarrow(A \vee A) \\
& \text { t3. }[A \vee(B \vee C)] \leftrightarrow[(A \vee B) \vee C] \\
& \text { t4. }[A \vee(B \wedge C)] \leftrightarrow[(A \vee B) \wedge(A \vee C)]
\end{aligned}
$$

Proof. The rules Trans and ECQ are immediate from their respective disjunctive versions. Then, $\mathrm{E} \vee, \mathrm{I} \wedge$, Sum, $\mathrm{t} 1, \mathrm{t} 2, \mathrm{t} 3$ and $\mathrm{t} 4$ are provable in the positive fragment of Anderson and Belnap's First Degree Entailment logic, FDE (cf. [1], pp. 158, ff.). (Mention to the use of MP has been omitted.)

We recall that $\mathrm{FDE}_{+}$is axiomatized with $A \rightarrow A(\mathrm{~A} 0), \mathrm{A} 1, \mathrm{~A} 3, \mathrm{~A} 5$, Trans and $\mathrm{A} 2$ and $\mathrm{A} 4$ in rule form (i.e., $A \rightarrow B \quad \& \quad A \rightarrow C \Rightarrow A \rightarrow(B \wedge C)$ and $A \rightarrow B \quad \& B \rightarrow C \Rightarrow(A \vee B) \rightarrow C)$, respectively). For axiomatizing FDE, we add $\mathrm{A} 8$ and the rule Contraposition (Con), $A \rightarrow B \Rightarrow \neg B \rightarrow \neg A$ (notice that $\mathrm{A} 0$ is then derivable as it is the case in $\mathrm{b}^{3}$ ).

Next, the logics $b_{1}^{3}, b_{2}^{3}$ are defined. They are two mutually independent extensions of $\mathrm{b}^{3}$. The former is contained in 2 of the 6 natural implicative expansions of K3; the latter, in the remaining 4 expansions. We have tried to axiomatize all systems with a common base as wide as possible.

Definition 5.3 (The basic logic $\mathbf{b}_{1}^{3}$ ) The logic $b_{1}^{3}$ is axiomatized by adding to $b^{3}$ the following axioms:

A9. $[(A \rightarrow B) \wedge A] \rightarrow B$

A10. $B \rightarrow(A \rightarrow B)$

A11. $A \vee(A \rightarrow B)$ 
Definition 5.4 (The basic logic $\mathbf{b}_{2}^{3}$ ) The logic $b_{2}^{3}$ is the result of adding the following axioms to $b^{3}$ :

$$
\begin{aligned}
& \text { A12. }[(A \rightarrow B) \wedge A] \rightarrow(\neg A \vee B) \\
& \text { A13. }[(A \rightarrow B) \wedge \neg B] \rightarrow(\neg A \vee B) \\
& \text { A14. } \neg A \rightarrow[A \vee(A \rightarrow B)] \\
& \text { A15. } B \rightarrow[\neg B \vee(A \rightarrow B)] \\
& \text { A16. }(A \vee \neg B) \vee(A \rightarrow B)
\end{aligned}
$$

Next, we prove some properties of $\mathrm{b}^{3}$ and its extensions. We begin by defining the notion of $a b^{3}$-theory and the classes of $b^{3}$-theories of interest in the present paper (recall that by EL, we generally refer to an extension (or an expansion, as the case may be) of the logic L; cf. Definition 2.3).

Definition 5.5 ( $\mathbf{E b}^{3}$-theories) Let $L$ be an $E b^{3}$-logic. An L-theory is a set of wffs closed under Adjunction (Adj) and provable L-entailment (L-ent). That is, $\mathcal{T}$ is an L-theory iff we have (1) whenever $A, B \in \mathcal{T}, A \wedge B \in \mathcal{T}$ (Adj); (2) whenever $A \rightarrow B$ is a theorem of $L$ and $A \in \mathcal{T}$, then $B \in \mathcal{T}$ (L-ent).

Definition 5.6 (Classes of $\mathbf{E b}^{3}$-theories) Let $L$ be an $E b^{3}$-logic and $\mathcal{T}$ an $L$-theory. We set (1) $\mathcal{T}$ is prime iff whenever $A \vee B \in \mathcal{T}$, then $A \in \mathcal{T}$ or $B \in \mathcal{T}$; (2) $\mathcal{T}$ is regular iff $\mathcal{T}$ contains all theorems of $L$; (3) $\mathcal{T}$ is inconsistent iff $A \wedge \neg A \in \mathcal{T}$ for some wff $A$ ( $\mathcal{T}$ is consistent iff it is not inconsistent).

Then, we have:

Proposition $5.7\left(\mathbf{E b}^{3}\right.$-theories and double negation) Let $L$ be an $E b^{3}$-logic and $\mathcal{T}$ an L-theory. Then, $A \in \mathcal{T}$ iff $\neg \neg A \in \mathcal{T}$.

Proof. Immediate by A8 (the use of L-ent is omitted in this and the proofs that follow).

Proposition 5.8 (Conjunction and disjunction in prime $\mathbf{E b}^{3}$-theories) Let $L$ be an $E b^{3}$-logic and $\mathcal{T}$ be an $L$-theory. Then, (1) $A \wedge B \in \mathcal{T}$ iff $A \in \mathcal{T}$ and $B \in \mathcal{T}$; (2) $\neg(A \wedge B) \in \mathcal{T} \quad$ iff $\neg A \in \mathcal{T}$ or $\neg B \in \mathcal{T}$; (3) $A \vee B \in \mathcal{T}$ iff $A \in \mathcal{T}$ or $B \in \mathcal{T} ;(4) \neg(A \vee B) \in \mathcal{T} \quad$ iff $\neg A \in \mathcal{T}$ and $\neg B \in \mathcal{T}$.

Proof. Case 1: by A1 and the fact that $\mathcal{T}$ is closed under Adj; case 2: by A3 and A7; case 3: by $\mathrm{A} 3$ and the fact that $\mathcal{T}$ is prime; case 4 : by $\mathrm{A} 6$ and the fact that $\mathcal{T}$ is closed under Adj.

Next, we remark some properties of $\mathrm{Eb}_{1}^{3}$ and $\mathrm{Eb}_{2}^{3}$-theories.

Proposition 5.9 (The conditional in prime regular $\mathbf{E b}_{1}^{3}$-theories) Let $L$ be an $E b_{1}^{3}$-logic and $\mathcal{T}$ be a prime regular $L$-theory. Then, $A \rightarrow B \in \mathcal{T}$ iff $A \notin \mathcal{T}$ or $B \in \mathcal{T}$. 
Proof. (a) From left to right $(\Rightarrow)$ : Immediate by A9. From right to left $(\Leftarrow)$ : Suppose $A \notin \mathcal{T}$. By A11 and regularity of $\mathcal{T}, A \vee(A \rightarrow B) \in \mathcal{T}$. Then, $A \rightarrow B \in \mathcal{T}$ by primeness. Suppose now $B \in \mathcal{T}$. Then, $A \rightarrow B \in \mathcal{T}$ is immediate by A10.

Proposition 5.10 (The cond. in prime regular consistent $\mathbf{E b}_{2}^{3}$-theories) Let $L$ be an $E b_{2}^{3}$-logic and $\mathcal{T}$ be a prime regular and consistent $L$-theory. Then, $A \rightarrow B \in \mathcal{T} \quad$ iff $\neg A \in \mathcal{T}$ or $B \in \mathcal{T}$ or $(A \notin \mathcal{T}$ and $\neg B \notin \mathcal{T})$.

Proof. (a) ( $\Rightarrow$ ): Suppose (1) $A \rightarrow B \in \mathcal{T}$ and, for reductio, (2) $\neg A \notin \mathcal{T}$ and $B \notin \mathcal{T}$ and $A \in \mathcal{T}$ or $(3) \neg A \notin \mathcal{T}$ and $B \notin \mathcal{T}$ and $\neg B \in \mathcal{T}$. Consider case (2): By Adj, we have (4) $A \rightarrow B \wedge A \in \mathcal{T}$, whence by A12, (5) $\neg A \vee B \in \mathcal{T}$, contradicting 2 by primeness of $\mathcal{T}$. Then, case (3) follows similarly by using A13. (b) $(\Leftarrow)$ : Suppose $(1) \neg A \in \mathcal{T}$ or $(2) B \in \mathcal{T}$ or $(3) A \notin \mathcal{T}$ and $\neg B \notin \mathcal{T}$. We have to prove $A \rightarrow B \in \mathcal{T}$. Consider case (1). By A14, $A \vee(A \rightarrow B) \in \mathcal{T}$ is deducible whence $A \rightarrow B \in \mathcal{T}$ follows by consistency and primeness of $\mathcal{T}$. Then, case (2) is provable in a similar way by using A15. Finally, case (3) follows by A16 $((A \vee \neg B) \vee(A \rightarrow B) \in \mathcal{T})$ and primeness and regularity of $\mathcal{T}$.

\section{Extension and primeness lemmas}

Firstly, we set a preliminary definition (cf. [7], pp. 24-25; cf. also [18], Chap. $4)$.

Definition 6.1 (Disjunctive $\mathbf{E b}^{3}$-derivability) Let $L$ be an $E b^{3}$-logic. For any sets of wffs $\Gamma, \Theta, \Theta$ is disjunctively derivable from $\Gamma$ in $L$ (in symbols, $\left.\Gamma \vdash_{L}^{d} \Theta\right)$ iff $A_{1} \wedge, \ldots \wedge A_{n} \vdash_{L} B_{1} \vee \ldots \vee B_{m}$ for some wffs $A_{1}, \ldots, A_{n} \in \Gamma$ and $B_{1}, \ldots, B_{m} \in \Theta$.

Next, we prove a lemma which is essential in order to prove the extension to maximal sets lemma. (In the rest of the section the subscript $\mathrm{Eb}^{3}$ (or L) is, in general, dropped from $\vdash_{\mathrm{Eb}^{3} \text { (or L) }}$ since no confusion can arise as we are speaking in general of $\mathrm{Eb}^{3}$-logics.)

Lemma 6.2 (Preliminary lemma to the extension lemma) Let $L$ be an $E b^{3}$-logic whose primitive rules of inference are in the set $\{A d j, M P, d M P$, $d$ Trans, $d E c q\}$. For any wffs $A, B_{1}, \ldots, B_{n}$, if $\left\{B_{1}, \ldots, B_{n}\right\} \vdash_{L} A$, then, for any wff $C, C \vee\left(B_{1} \wedge \ldots \wedge B_{n}\right) \vdash_{L} C \vee A$.

Proof. (Cf. [6], p. 27) Induction on the length of the proof of $A$ from $\left\{B_{1}, \ldots, B_{n}\right\}$ (H.I abbreviates hypothesis of induction). (1) $A \in\left\{B_{1}, \ldots, B_{n}\right\}$. Let $A$ be $B_{i}(1 \leq i \leq n)$. By elementary properties of $\wedge, \vdash\left(B_{1} \wedge \ldots \wedge B_{n}\right) \rightarrow B_{i}$. By $\operatorname{Sum}(A \rightarrow B \Rightarrow(C \vee A) \rightarrow(C \vee B)), C \vee\left(B_{1} \wedge \ldots \wedge B_{n}\right) \vdash C \vee A$. (2) $A$ is an axiom. By A3, $\vdash C \vee A$. So, $C \vee\left(B_{1} \wedge \ldots \wedge B_{n}\right) \vdash C \vee A$. (3) $A$ is by Adj. Then, $A$ is $D \wedge E$ for some wffs $D$ and $E$. By H.I, $C \vee\left(B_{1} \wedge \ldots \wedge B_{n}\right) \vdash C \vee D$ and 
$C \vee\left(B_{1} \wedge \ldots \wedge B_{n}\right) \vdash C \vee E$ whence $C \vee\left(B_{1} \wedge \ldots \wedge B_{n}\right) \vdash(C \vee D) \wedge(C \vee E)$ by Adj. Finally, $C \vee\left(B_{1} \wedge \ldots \wedge B_{n}\right) \vdash C \vee(D \wedge E)$ by t4 $([A \vee(B \wedge C)] \leftrightarrow[(A \vee B) \wedge(C \vee D)])$. (4) $A$ is by MP. By H.I, $C \vee\left(B_{1} \wedge \ldots \wedge B_{n}\right) \vdash C \vee(D \rightarrow A)$ and $C \vee\left(B_{1} \wedge \ldots \wedge B_{n}\right) \vdash C \vee D$ for some wff $D$. So, $C \vee\left(B_{1} \wedge \ldots \wedge B_{n}\right) \vdash C \vee A$ by dMP. Now, the cases of the disjunctive rules are proved similarly. For instance, let us prove the case when $A$ is by dMP. Then, $A$ is $D \vee E$ for some wffs $D$ and $E$. By H.I, $C \vee\left(B_{1} \wedge \ldots \wedge B_{n}\right) \vdash$ $C \vee(D \vee F)$ and $C \vee\left(B_{1} \wedge \ldots \wedge B_{n}\right) \vdash C \vee[D \vee(F \rightarrow E)]$ for some wff $F$, whence $C \vee\left(B_{1} \wedge \ldots \wedge B_{n}\right) \vdash(C \vee D) \vee F$ and $C \vee\left(B_{1} \wedge \ldots \wedge B_{n}\right) \vdash(C \vee D) \vee(F \rightarrow E)$ by t3 $([A \vee(B \vee C)] \leftrightarrow[(A \vee B) \vee C])$. So, $C \vee\left(B_{1} \wedge \ldots \wedge B_{n}\right) \vdash(C \vee D) \vee E$ by dMP, and finally, $C \vee\left(B_{1} \wedge \ldots \wedge B_{n}\right) \vdash C \vee(D \vee E)$ by t3, as it was required. The cases where $A$ is by dTrans and dECQ are proved similarly.

Now, we proceed to show how to extend sets of wffs to maximal sets (cf. Lemma 9 in [7] and Chapter 4 in [18]).

Definition 6.3 (Maximal sets) Let $L$ be an $E b^{3}$-logic. $\Gamma$ is an L-maximal set of wffs iff $\Gamma \nvdash_{L}^{d} \bar{\Gamma} \bar{\Gamma}$ is the complement of $\left.\Gamma\right)$.

Lemma 6.4 (Extension to maximal sets) Let $L$ be an $E b^{3}$-logic whose primitive rules of inference are in the set $\{A d j, M P, d M P, d$ Trans, $d E c q\}$ and let $\Gamma$, $\Theta$ be sets of wffs such that $\Gamma \nvdash_{L}^{d} \Theta$. Then, there are sets of wffs $\Gamma^{\prime}, \Theta^{\prime}$ such that $\Gamma \subseteq \Gamma^{\prime}, \Theta \subseteq \Theta^{\prime}, \Theta^{\prime}=\bar{\Gamma}^{\prime}$ and $\Gamma^{\prime} \nvdash_{L}^{d} \Theta^{\prime}$ (that is, $\Gamma^{\prime}$ is an L-maximal set such that $\left.\Gamma^{\prime} \nvdash_{L}^{d} \Theta^{\prime}\right)$.

Proof. Let $A_{1}, \ldots, A_{n}, \ldots$, be an enumeration of the wffs. The sets $\Gamma^{\prime}$ and $\Theta^{\prime}$ are defined as follows: $\Gamma^{\prime}=\bigcup_{k \in \mathbb{N}} \Gamma_{k}, \Theta^{\prime}=\bigcup_{k \in \mathbb{N}} \Theta_{k}$ where $\Gamma_{0}=\Gamma, \Theta_{0}=\Theta$ and for each $k \in \mathbb{N}, \Gamma_{k+1}$ and $\Theta_{k+1}$ are defined as follows: (i) if $\Gamma_{k} \cup\left\{A_{k+1}\right\} \vdash^{d} \Theta_{k}$, then $\Gamma_{k+1}=\Gamma_{k}$ and $\Theta_{k+1}=\Theta_{k} \cup\left\{A_{k+1}\right\}$; (ii) if $\Gamma_{k} \cup\left\{A_{k+1}\right\} \nvdash^{d} \Theta_{k}$, then $\Gamma_{k+1}=\Gamma_{k} \cup\left\{A_{k+1}\right\}$ and $\Theta_{k+1}=\Theta_{k}$. Notice that $\Gamma \subseteq \Gamma^{\prime}, \Theta \subseteq \Theta^{\prime}$ and that $\Gamma^{\prime} \cup \Theta^{\prime}=\mathcal{F}$. We prove (I) $\Gamma_{k} \nvdash^{d} \Theta_{k}$ for all $k \in \mathbb{N}$. We proceed by reductio ad absurdum. So, suppose that for some $i \in \mathbb{N}$, (II) $\Gamma_{i} \nvdash^{d} \Theta_{i}$ but $\Gamma_{i+1} \vdash^{d} \Theta_{i+1}$. We then consider the two possibilities (i) and (ii) above according to which $\Gamma_{i+1}$ and $\Theta_{i+1}$ are defined: (a) $\Gamma_{i} \cup\left\{A_{i+1}\right\} \nvdash^{d} \Theta_{i}$. By (ii), $\Gamma_{i+1}=\Gamma_{i} \cup\left\{A_{i+1}\right\}$ and $\Theta_{i+1}=\Theta_{i}$. By the reductio hypothesis (II), $\Gamma_{i} \cup\left\{A_{i+1}\right\} \vdash^{d} \Theta_{i}$, a contradiction. (b) $\Gamma_{i} \cup\left\{A_{i+1}\right\} \vdash^{d} \Theta_{i}$. By (i), $\Gamma_{i+1}=\Gamma_{i}$ and $\Theta_{i+1}=\Theta_{i} \cup\left\{A_{i+1}\right\}$. By the reductio hypothesis (II), (1) $\Gamma_{i} \vdash^{d} \Theta_{i} \cup\left\{A_{i+1}\right\}$. Now, let the formulas of $\Gamma_{i}$ and $\Theta_{i}$ in this derivation be $B_{1}, \ldots, B_{m}$ and $C_{1}, \ldots, C_{n}$, respectively, and let us refer by $B$ to $B_{1} \wedge \ldots \wedge B_{n}$ and by $C$ to $C_{1} \vee \ldots \vee C_{n}$. Then (1) can be rephrased as follows (2) $B \vdash C \vee A_{i+1}$. On the other hand, given the hypothesis (b), there is a conjunction $B^{\prime}$ of elements of $\Gamma_{i}$ and some disjunction $C^{\prime}$ of elements of $\Theta_{i}$ such that (3) $B^{\prime} \wedge A_{i+1} \vdash C^{\prime}$. Let us now refer by $B^{\prime \prime}$ to $B \wedge B^{\prime}$ and by $C^{\prime \prime}$ to $C \vee C^{\prime}$; we will show (III) $B^{\prime \prime} \vdash C^{\prime \prime}$, that is, $\Gamma_{i} \vdash^{d} \Theta_{i}$, contradicting the reductio hypothesis and thus proving (I). By elementary properties of $\wedge$ and $\vee$, we have (4) $B^{\prime \prime} \wedge A_{i+1} \vdash C^{\prime \prime}$ from (3), and (5) $B^{\prime \prime} \vdash C^{\prime \prime} \vee A_{i+1}$ from (2). By (5), we get

(6) $B^{\prime \prime} \vdash C^{\prime \prime} \vee\left(B^{\prime \prime} \wedge A_{i+1}\right)$ and by (4) and Lemma 6.2, (7) $C^{\prime \prime} \vee\left(B^{\prime \prime} \wedge A_{i+1}\right)$ $\vdash C^{\prime \prime} \vee C^{\prime \prime}$ whence by t2 $(A \leftrightarrow(A \vee A))$, we have (8) $C^{\prime \prime} \vee\left(B^{\prime \prime} \wedge A_{i+1}\right) \vdash C^{\prime \prime}$. By 
(6) and (8) we get (III) $B^{\prime \prime} \vdash C^{\prime \prime}$, that is, $\Gamma_{i} \vdash^{d} \Theta_{i}$, contradicting the reductio hypothesis. Consequently, (I) $\left(\Gamma_{k} \nvdash^{d} \Theta_{k}\right.$ for all $\left.k \in \mathbb{N}\right)$ is proved. Thus, we have sets of wffs $\Gamma^{\prime}, \Theta^{\prime}$ such that $\Gamma \subseteq \Gamma^{\prime}, \Theta \subseteq \Theta^{\prime}, \Gamma^{\prime} \nvdash^{d} \Theta^{\prime}$ (since $\Gamma_{k} \nvdash^{d} \Theta_{k}$ for all $k \in \mathbb{N}$ ) and $\Theta^{\prime}=\bar{\Gamma}^{\prime}$ (since $\Gamma^{\prime} \cap \Theta^{\prime}=\emptyset$ - otherwise $\Gamma_{i} \vdash^{d} \Theta_{i}$ for some $i \in \mathbb{N}$ and $\Gamma^{\prime} \cup \Theta^{\prime}=\mathcal{F}$ ), as it was required. Finally, notice that $\Gamma^{\prime}$ is maximal (since $\left.\Gamma^{\prime} \nvdash^{d} \bar{\Gamma}\right)$.

Before proving the primeness lemma we pause a second to remark the essential role Lemma 6.2 has played in the proof of the extension lemma just given (notice that the rest of syntactical moves required in the said proof can be carried on by leaning on the simple resources of the positive fragment of FDE - cf. the proof of Proposition 5.2).

Lemma 6.5 (Primeness) Let $L$ be an $E b^{3}$-logic whose primitive rules of inference are in the set $\{A d j, M P, d M P$, dTrans, dEcq $\}$. If $\Gamma$ is a L-maximal set, then it is a prime L-theory closed under the rules of $L$.

Proof. (Cf. Lemma 8 in [7]) (1) $\Gamma$ is a theory: It is trivial to prove that $\Gamma$ is a theory closed under the rules of $b^{3}$. For example, let us prove that $\Gamma$ is closed under dTrans. For reductio, suppose that there are wffs $A, B, C, D$ such that $D \vee(A \rightarrow B) \in \Gamma, D \vee(B \rightarrow C) \in \Gamma$ but $D \vee(A \rightarrow C) \notin \Gamma$. Then, $[D \vee(A \rightarrow B)] \wedge[D \vee(B \rightarrow C)] \vdash D \vee(A \rightarrow B)$ and $[D \vee(A \rightarrow B)] \wedge[D \vee(B \rightarrow$ $C)] \vdash D \vee(B \rightarrow C)$, whence $[D \vee(A \rightarrow B)] \wedge[D \vee(B \rightarrow C)] \vdash D \vee(A \rightarrow C)$ by dTrans, contradicting the maximality of $\Gamma$. (2) $\Gamma$ is prime: If there are some wffs $A, B$ such that $A \vee B \in \Gamma$ but $A \notin \Gamma$ and $B \notin \Gamma$, then $\Gamma$ is not maximal by virtue of $\mathrm{t} 1((A \vee B) \rightarrow(A \vee B))$.

\section{Extensions of the basic logics}

In this section, 6 different extensions of $\mathrm{b}^{3}$ are defined. It will be proved that each one of the 6 natural implicative expansions of MK3 defined in Proposition 4.4 characterizes or determines one of these extensions. Two of these extensions of $b^{3}$ contain $b_{1}^{3}$ while $b_{2}^{3}$ is contained in the remaining four systems.

The extensions just referred to are axiomatized using the following list of axioms:

$$
\begin{aligned}
& \text { A17. } \neg(A \rightarrow B) \rightarrow(A \wedge \neg B) \\
& \text { A18. }(A \wedge \neg B) \rightarrow \neg(A \rightarrow B) \\
& \text { A19. } A \rightarrow[B \vee \neg(A \rightarrow B)] \\
& \text { A20. } \neg B \rightarrow[\neg A \vee \neg(A \rightarrow B)] \\
& \text { A21. }[\neg(A \rightarrow B) \wedge \neg A] \rightarrow A \\
& \text { A22. } \neg(A \rightarrow B) \rightarrow(A \vee \neg B) \\
& \text { A23. }(\neg A \wedge B) \rightarrow(A \rightarrow B) \\
& \text { A24. }[\neg(A \rightarrow B) \wedge B] \rightarrow \neg B
\end{aligned}
$$




$$
\begin{aligned}
& \text { A25. }(A \wedge \neg B) \rightarrow[\neg A \vee \neg(A \rightarrow B)] \\
& \text { A26. } \neg(A \rightarrow B) \rightarrow \neg B \\
& \text { A27. } \neg(A \rightarrow B) \rightarrow A
\end{aligned}
$$

Definition 7.1 (Extensions of $\mathbf{b}_{1}^{3}$ ) The two extensions of $b_{1}^{3}$ are Lt1 and Lt2. It will be proved that the logic Lti is characterized by the matrix Mti $(i \in\{1,2\})$. These logics are axiomatized by adding the following axioms to $b_{1}^{3}$.

Lt1:

$$
\begin{aligned}
& \text { A17. } \neg(A \rightarrow B) \rightarrow(A \wedge \neg B) \\
& \text { A18. }(A \wedge \neg B) \rightarrow \neg(A \rightarrow B)
\end{aligned}
$$

Lt2:

$$
\text { A19. } A \rightarrow[B \vee \neg(A \rightarrow B)]
$$

Definition 7.2 (Extensions of $\mathbf{b}_{2}^{3}$ ) The four extensions of $b_{2}^{3}$ are Lt3, Lt4, Lt5 and Lt6. As in the case of the extensions of $b_{1}^{3}$, it will be proved that the logic Lti is characterized by the matrix Mti $(i \in\{3,4,5,6\})$. These four extensions of $b_{2}^{3}$ are axiomatized by adding the following axioms to $b_{2}^{3}$ :

Lt3:

$$
\begin{aligned}
& \text { A19. } A \rightarrow[B \vee \neg(A \rightarrow B)] \\
& \text { A20. } \neg B \rightarrow[\neg A \vee \neg(A \rightarrow B)] \\
& \text { A21. }(\neg(A \rightarrow B) \wedge \neg A] \rightarrow A \\
& \text { A22. } \neg(A \rightarrow B) \rightarrow(A \vee \neg B) \\
& \text { A23. }(\neg A \wedge B) \rightarrow(A \rightarrow B) \\
& \text { A24. }[\neg(A \rightarrow B) \wedge B] \rightarrow \neg B
\end{aligned}
$$

Lt4:

$$
\begin{aligned}
& \text { A17. } \neg(A \rightarrow B) \rightarrow(A \wedge \neg B) \\
& A 25 .(A \wedge \neg B) \rightarrow[\neg A \vee \neg(A \rightarrow B)]
\end{aligned}
$$

Lt5:

$$
\begin{aligned}
& \text { A20. } \neg B \rightarrow[\neg A \vee \neg(A \rightarrow B) \\
& \text { A21. }[\neg(A \rightarrow B) \wedge \neg A] \rightarrow A \\
& \text { A26. } \neg(A \rightarrow B) \rightarrow \neg B
\end{aligned}
$$

Lt6:

$$
\begin{aligned}
& \text { A19. } A \rightarrow[B \vee \neg(A \rightarrow B)] \\
& \text { A24. }[\neg(A \rightarrow B) \wedge B] \rightarrow \neg B \\
& \text { A27. } \neg(A \rightarrow B) \rightarrow A
\end{aligned}
$$


In what follows, we prove two important propositions on the behavior of negated conditionals in the extensions of $b_{1}^{3}$ and $b_{2}^{3}$ just defined. Notice that Lt4 is (an axiomatization of) Lukasiewicz's 3-valued logic L3 (cf. [17] and the references therein). On the other hand, Lt5 is the logic G3 $3_{\mathrm{L}}^{1}$, related to Gödelian 3 -valued logic G3, studied in [15].

Proposition 7.3 (Negated conditionals in $\mathbf{E b}_{1}^{3}$-logics) Let $L$ be an ELtilogic where Lti will refer in each case to one of the extensions of $b_{1}^{3}$ displayed in Definition 7.1. And let $\mathcal{T}$ be a prime, regular and consistent L-theory. We have:

- ELt1-logics: $\neg(A \rightarrow B) \in \mathcal{T}$ iff $A \in \mathcal{T} \quad \& \neg B \in \mathcal{T}$.

- ELt2-logics: $\neg(A \rightarrow B) \in \mathcal{T}$ iff $A \in \mathcal{T} \quad \& \quad B \notin \mathcal{T}$.

Proposition 7.4 (Negated conditionals in $\mathbf{E b}_{2}^{3}$-logics) Let $L$ be an ELtilogic where Lti will refer in each case to one of the extensions of $b_{2}^{3}$ displayed in Definition 7.2. And let $\mathcal{T}$ be a prime, regular and consistent L-theory. We have:

- ELt3-logics: $\neg(A \rightarrow B) \in \mathcal{T}$ iff $(A \in \mathcal{T} \quad \& B \notin \mathcal{T})$ or $(\neg A \notin \mathcal{T} \& \neg B \in$ $\mathcal{T})$.

- ELt4-logics: $\neg(A \rightarrow B) \in \mathcal{T}$ iff $A \in \mathcal{T} \quad \& \neg B \in \mathcal{T}$.

- ELt5-logics: $\neg(A \rightarrow B) \in \mathcal{T}$ iff $\neg A \notin \mathcal{T} \quad \& \neg B \in \mathcal{T}$.

- ELt6-logics: $\neg(A \rightarrow B) \in \mathcal{T}$ iff $A \in \mathcal{T} \quad \& B \notin \mathcal{T}$.

The proof of Propositions 7.3 and 7.4 is similar to that of 5.9 and 5.10. So, it will suffice to prove a couple of cases.

Proof. Proposition 7.3. ELt1-logics. The equivalence $\neg(A \rightarrow B) \in \mathcal{T}$ iff $A \in \mathcal{T}$ and $\neg B \in \mathcal{T}$ follows immediately by A17 and A18.

Proposition 7.4. ELt3-logics.

(a) $(\Rightarrow)$ Suppose $(1) \neg(A \rightarrow B) \in \mathcal{T}$ and, for reductio, $(A \notin \mathcal{T}$ or $B \in \mathcal{T})$ and $(\neg A \in \mathcal{T}$ or $\neg B \notin \mathcal{T})$. There are four cases to consider:
1. $A \notin \mathcal{T} \quad \& \neg A \in \mathcal{T}$
2. $A \notin \mathcal{T} \& \neg B \notin \mathcal{T}$
3. $B \in \mathcal{T} \& \neg A \in \mathcal{T}$
4. $B \in \mathcal{T} \& \neg B \notin \mathcal{T}$

Then, cases 1, 3 and 4 follow immediately by A21, A23 and A24, respectively, while case 2 is derivable by $\mathrm{A} 22$ and primeness of $\mathcal{T}$.

(b) $(\Leftarrow)$ Suppose $(1)(A \in \mathcal{T} \quad \& \quad B \notin \mathcal{T})$ or $(2)(\neg A \notin \mathcal{T} \quad \& \quad \neg B \in \mathcal{T})$. Then, $\neg(A \rightarrow B) \in \mathcal{T}$ follows by (1) and A19, and from (2) and A20. 


\section{Belnap-Dunn semantics for the Lt $i$-logics}

We will define two types of models. Models for extensions of $b_{1}^{3}$ or $\mathrm{Eb}_{1}^{3}$-models, and models for extensions of $b_{2}^{3}$ or $\mathrm{Eb}_{2}^{3}$-models. The two types of models are distinguished by different necessary and sufficient conditions for assigning truth and falsity to the conditionals. In particular, we have:

Definition 8.1 (Eb-models) An Eb $b_{1}^{3}$-model is a structure $(K, I)$ where $(i)$ $K=\{\{T\},\{F\}, \emptyset\}$ and (ii) $I$ is an $E b_{1}^{3}$-interpretation from the set of all wffs to $K$, this notion being defined according to the following conditions for each propositional variable $p$ and wffs $A, B:$ (1) $I(p) \in K$; (2a) $T \in I(\neg A)$ iff $F \in I(A)$; (2b) $F \in I(\neg A)$ iff $T \in I(A)$; (3a) $T \in I(A \wedge B)$ iff $T \in I(A)$ and $T \in I(B)$; (3b) $F \in I(A \wedge B)$ iff $F \in I(A)$ or $F \in I(B)$; (4a) $T \in I(A \vee B)$ iff $T \in I(A)$ or $T \in I(B)$; (4b) $F \in I(A \vee B)$ iff $F \in I(A)$ and $F \in I(B)$; (5a) $T \in I(A \rightarrow B)$ iff $T \notin I(A)$ or $T \in I(B)$; (5b) There are two possibilities for assigning $F$ to conditionals: $\left(5 b_{1}\right) F \in I(A \rightarrow B)$ iff $T \in I(A)$ and $F \in I(B)$; and $\left(5 b_{2}\right) F \in I(A \rightarrow B)$ iff $T \in I(A)$ and $T \notin I(B)$.

Definition 8.2 (Eb-models) An Eb $b_{2}^{3}$-model is a structure $(K, I)$ where $K$ and $I$ are defined similarly as in E $b_{1}^{3}$-models, save for clauses (5a) and (5b), which are now as follows. (5a) $T \in I(A \rightarrow B)$ iff $F \in I(A)$ or $T \in I(B)$ or $(T \notin I(A)$ and $F \notin I(B))$; (5b) There are four possibilities for assigning $F$ to conditionals: $\left(5 b_{1}\right) F \in I(A \rightarrow B)$ iff $(T \in I(A)$ and $T \notin I(B))$ or $(F \notin I(A)$ and $F \in I(B)) ;\left(5 b_{2}\right) F \in I(A \rightarrow B)$ iff $T \in I(A)$ and $F \in I(B) ;\left(5 b_{3}\right)$ $F \in I(A \rightarrow B)$ iff $F \notin I(A)$ and $F \in I(B) ;\left(5 b_{4}\right) F \in I(A \rightarrow B)$ iff $T \in I(A)$ and $T \notin I(B)$.

Then, Lt $i$-models are as follows:

Definition 8.3 (Lt $i$-models) Lti-models are:

- Lt1-models: Lt1-models are Eb $b_{1}^{3}$-models with clause (5b1).

- Lt2-models: Lt2-models are Eb $b_{1}^{3}$-models with clause (5b2).

- Lt3-models: Lt3-models are Eb $b_{2}^{3}$-models with clause (5b1).

- Lt4-models: Lt4-models are Eb $b_{2}^{3}$-models with clause (5b2).

- Lt5-models: Lt5-models are E $b_{2}^{3}$-models with clause (5b3).

- Lt6-models: Lt6-models are Eb $b_{2}^{3}$-models with clause (5b4).

Finally, the general notions of Lt $i$-consequence and Lt $i$-validity are defined as in K3-models (cf. Definition 3.3).

Definition 8.4 (Lt $i$-consequence, Lt $i$-validity) Let $M$ be an Lti-model $(1 \leq$ $i \leq 6)$. For any set of wffs $\Gamma$ and wff $A, \Gamma \vDash_{M} A$ ( $A$ is a consequence 
of $\Gamma$ in the Lti-model $M)$ iff $T \in I(A)$ whenever $T \in I(\Gamma)[T \in I(\Gamma)$ iff $\forall A \in \Gamma(T \in I(A)) ; F \in I(\Gamma)$ iff $\exists A \in \Gamma(F \in I(A))]$. Then, $\Gamma \vDash_{L t i} A$ (A is a consequence of $\Gamma$ in Lti-semantics) iff $\Gamma \vDash_{M} A$ for each Lti-model M. In particular, $\vDash_{L t i} A$ ( $A$ is valid in Lti-semantics) iff $\vDash_{M}$ A for each Lti-model $M$ (i.e., iff $T \in I(A)$ for each Lti-model $M)$. (By $\vDash_{L t i}$ we shall refer to the relation just defined.)

Next, it is proved that the consequence relation $\vDash_{\mathrm{Mt} i}$ (cf. Proposition 4.4 and Definition 3.1) and the relation $\vDash_{\mathrm{Lt} i}$ just defined are coextensive. Then, soundness of each Lt $i$-logic w.r.t. both consequence relations will immediately follow.

Proposition 8.5 (Coextensiveness of $\vDash_{\mathrm{Mt} i}$ and $\left.\vDash_{\mathrm{Lt} i}\right)$ For any $i(1 \leq i \leq$ $6)$, set of wffs $\Gamma$ and wff $A, \Gamma \vDash_{M t i} A$ iff $\Gamma \vDash_{L t i} A$. In particular, $\vDash_{M t i} A$ iff $\vDash_{L t i} A$.

Proof. It is similar to the corresponding proposition in [16], Proposition $7.4^{6}$.

Now, we can prove soundness.

Theorem 8.6 (Soundness of Lt $i$ w.r.t. $\vDash_{\mathbf{M t} i}$ and $\left.\vDash_{\mathbf{L t} i}\right)$ For any $i(1 \leq i \leq$ 6), set of wffs $\Gamma$ and wff $A$, if $\Gamma \vdash_{L t i} A$, then (1) $\Gamma \vDash_{M t i} A$ and (2) $\Gamma \vDash_{L t i} A$.

Proof. (1) Given a particular logic Lti, it is easy to check that the rules Adj, MP, dMP, dTrans and dECQ preserve Mti-validity, whereas the axioms of Lt $i$ are assigned 2 by any Mti-interpretation $I$. Consequently, if $\Gamma \vdash_{\mathrm{Lt} i} A$, then $\Gamma \vDash_{\mathrm{Mti}}$ A. (2). Then (2) is immediate by (1) and Proposition 8.5. Finally, if $\Gamma$ is the empty set, the proof is similar. (In case a tester is needed, the reader con use that in [10].)

\section{Completeness of the Lt $i$-logics}

We shall prove the completeness of Lt $i(1 \leq i \leq 6)$ (cf. Definitions 7.1 and 7.2) w.r.t. both $\vDash_{\mathrm{Mt} i}$ and $\vDash_{\mathrm{Lt} i}$.

Completeness w.r.t. $\vDash_{\mathrm{Lt} i}$ is proved by means of a canonical model construction. Then, completeness w.r.t. $\vDash_{\mathrm{Mt} i}$ follows immediately by Proposition 8.5.

We begin by the definition of canonical Lti-models. It will be proved that if $A$ is not derivable from $\Gamma$ in Lti, then $A$ does not follow from $\Gamma$ in some canonical Lt $i$-model. The concept of a canonical Lt $i$-model is based upon the notion of a $\mathcal{T}$-interpretation. In what follows, we refer by Lt $i(1 \leq i \leq 6)$ to any of the 6 extensions of $\mathrm{b}^{3}$ defined in section 7 .

Definition 9.1 ( $\mathcal{T}$-interpretations) Let $K$ be the set $\{\{T\},\{F\}, \emptyset\}$ as in Definition 8.1. And let $L$ be an ELti-logic and $\mathcal{T}$ be a prime, regular and consistent

\footnotetext{
${ }^{6}$ It has to be remarked that the appropriate equivalences are now the following: $\{T\}=2$; $\emptyset=1 ;$ and $\{F\}=0$.
} 
$L$-theory. Then, the function I from the set of all wffs to $K$ is defined as follows: for each propositional variable $p$, we set (a) $T \in I(p)$ iff $p \in \mathcal{T}$; (b) $F \in I(p)$ iff $\neg p \in \mathcal{T}$. Next, $I$ assigns a member of $K$ to each wff $A$ according to the corresponding conditions 2, 3, 4 and 5 in Definitions 8.1-8.3. Then, it is said that $I$ is a $\mathcal{T}$-interpretation. (As in Definition 8.4, $T \in I(\Gamma)$ iff $\forall A \in \Gamma(T \in I(A))$; $F \in I(\Gamma)$ iff $\exists A \in \Gamma(F \in I(A))$.)

Definition 9.2 (Canonical ELt $i$-models) A canonical ELti-model is a structure $\left(K, I_{\mathcal{T}}\right)$ where $K$ is defined as in Definition 8.1 and $I_{\mathcal{T}}$ is a $\mathcal{T}$-interpretation built upon a prime, regular and consistent $L$-theory $\mathcal{T}$ ( $L$ is an ELti-logic).

Definition 9.3 (The canonical relation $\vDash_{I_{\mathcal{T}}}$ ) Let $\left(K 3, I_{\mathcal{T}}\right)$ be a canonical Lti-model. The canonical relation $\vDash_{I_{\mathcal{T}}}$ is defined as follows. For any set of $w f f s$ $\Gamma$ and wff $A, \Gamma \vDash_{I_{\mathcal{T}}} A$ ( $A$ is a consequence of $\Gamma$ in the canonical Lti-model (K3, $\left.I_{\mathcal{T}}\right)$ ) iff $T \in I_{\mathcal{T}}(A)$ whenever $T \in I_{\mathcal{T}}(\Gamma)$. In particular, $\vDash_{I_{\mathcal{T}}} A$ (A is true in the canonical Lti-model $\left.\left(K 3, I_{\mathcal{T}}\right)\right)$ iff $T \in I_{\mathcal{T}}(A)$.

By Definitions 9.2 and 9.3, it is clear that any canonical Lt $i$-model is an Lt $i$-model.

Proposition 9.4 (Any canonical Lt $i$-model is an Lt $i$-model) Let $M=(K 3$, $I_{\mathcal{T}}$ ) be a canonical Lti-model. Then, $M$ is indeed an Lti-model.

Proof. It follows immediately by Definitions 9.2 and 9.3 (by the way, notice that each propositional variable - and so, each wff- can be assigned $\{T\},\{F\}$ or $\emptyset$ since $\mathcal{T}$, although consistent, is not required to be complete in the classical sense).

Given Proposition 9.4, Lemma 9.5 is the essential fact we have to prove in order to prove completeness. In this lemma, conditions (a) and (b) in Definition 9.1 are generalized to all wffs. The proof of Lemma 9.5 leans on Propositions $5.7,5.8,5.9,5.10,7.3$ and 7.4 .

Lemma 9.5 ( $\mathcal{T}$-interpreting the set of wffs) Let $L$ be an ELti-logic and $I$ be a $\mathcal{T}$-interpretation defined on the $L$-theory $\mathcal{T}$. For each wff $A$, we have: (1) $T \in I(A)$ iff $A \in \mathcal{T}$; (2) $F \in I(A)$ iff $\neg A \in \mathcal{T}$.

Proof. Induction on the length of $A$ (H.I. abbreviates "hypothesis of induction"). Clauses cited in (II) below refer to clauses in Definition 8.1 or Definition 8.2 .

(I) $A$ is a propositional variable, a conjunction, a disjunction or a negative formula. The proof, similar to that of Proposition 8.5 in [16], is easy and leans upon Propositions 5.7 and 5.8.

(II) $A$ is a conditional $B \rightarrow C$ assigned $T$ by I.

(IIi) Lt $i$ is an $\mathrm{Eb}_{1}^{3}$-logic: $T \in I(B \rightarrow C)$ iff (clause $5 \mathrm{a}$ in Definition 8.1) $T \notin$ $I(B)$ or $T \in I(C)$ iff (H.I.) $B \notin \mathcal{T}$ or $C \in \mathcal{T}$ iff (Proposition 5.9) $B \rightarrow C \in \mathcal{T}$. 
(IIii) Lt $i$ is an $\mathrm{Eb}_{2}^{3}$-logic: $T \in I(B \rightarrow C)$ iff (clause 5a in Definition 8.2) $(F \in I(B)$ or $T \in I(C))$ or $(T \notin I(B)$ and $F \notin I(C))$ iff (H.I.) $(\neg B \in \mathcal{T}$ or $C \in \mathcal{T})$ or $(B \notin \mathcal{T}$ and $\neg C \notin \mathcal{T})$ iff (Proposition 5.10) $B \rightarrow C \in \mathcal{T}$.

(III) $A$ is a conditional $B \rightarrow C$ assigned $F$ by $I$. We have to consider six different cases, but we think that a couple of examples will be sufficient.

(IIIi) Lt $i$ is an $\mathrm{Eb}_{1}^{3}$-logic: Let Lt $i$ be, for instance, Lt1. We have $F \in I(B \rightarrow$ $C$ ) iff (clause $5 b_{1}$ in Definition 8.3) $T \in I(B)$ and $F \in I(C)$ iff (H.I.) $B \in \mathcal{T}$ and $\neg C \in \mathcal{T}$ iff (Proposition 7.3) $\neg(B \rightarrow C) \in \mathcal{T}$.

(IIIii) Lt $i$ is an $\mathrm{Eb}_{2}^{3}$-logic: Let Lt $i$ be, for instance, Lt3. We have $F \in I(B \rightarrow$ $C)$ iff (clause $5 b_{1}$ in Definition 8.3) $(T \in I(B)$ and $T \notin I(C))$ or $(F \notin I(B)$ and $F \in I(C))$ iff (H.I.) $(B \in \mathcal{T}$ and $C \notin \mathcal{T})$ or $(\neg B \notin \mathcal{T}$ and $\neg C \in \mathcal{T})$ iff (Proposition 7.4) $\neg(B \rightarrow C) \in \mathcal{T}$.

Next, we recall the notion of set of consequences of a given set of formulas $\Gamma$ in Lt $i$ and then we prove completeness.

Definition 9.6 (The set of consequences of $\Gamma$ in Lt $i$ ) The set of consequences in Lti of a set of wffs $\Gamma$ (in symbols $C n \Gamma[L t i]$ ) is defined as follows: $C n \Gamma[L t i]=$ $\left\{A \mid \Gamma \vdash_{L t i} A\right\}$.

We note the following remark.

Remark 9.7 (The set of consequences of $\Gamma$ in Lt $i$ is a regular theory) It is obvious that for any $\Gamma, C n \Gamma[L t i]$ is closed under Adj, MP, dMP, dTrans and $d E C Q$ and contains all theorems of Lti. Consequently, it is closed under Lti-entailment.

Theorem 9.8 (Completeness of Lt $i$-logics) For any $i(1 \leq i \leq 6)$, set of wffs $\Gamma$ and wff $A$, (1) if $\Gamma \vDash_{L t i} A$, then $\Gamma \vdash_{L t i} A$; (2) if $\Gamma \vDash_{M t i} A$, then $\Gamma \vdash_{L t i}$ $A$.

Proof. (1) Suppose there are set of wffs $\Gamma$ and wff $A$ such that $\Gamma \nvdash_{\mathrm{Lt} i} A$. We prove $\Gamma \nvdash_{\mathrm{Lt} i} A$. If $\Gamma \nvdash_{\mathrm{Lt} i} A$, clearly $A \notin \mathrm{Cn} \Gamma[\mathrm{Lt} i]$. Thus, $\mathrm{Cn} \Gamma[\mathrm{Lt} i] \nvdash_{\mathrm{Lt} i}^{d}\{A\}$ : otherwise $\left\{B_{1} \wedge \ldots \wedge B_{n}\right\} \vdash_{\mathrm{Lt} i} A$ for some $B_{1}, \ldots, B_{n} \in \mathrm{Cn} \Gamma[\mathrm{Lt} i]$, whence $A$ would be in $\mathrm{Cn} \Gamma[\mathrm{Lt} i]$ after all. Then, by Lemma 6.4 , there is an Lti-maximal set $\mathcal{T}$ such that $\mathrm{Cn} \Gamma[$ Lti $i] \subseteq \mathcal{T}$ and $A \notin \mathcal{T}$. So, $\Gamma \subseteq \mathcal{T}$ (since $\Gamma \subseteq \mathrm{Cn} \Gamma[$ Lti $i]$ ) and $A \notin \mathcal{T}$. By Lemma 6.5, $\mathcal{T}$ is a prime Lti-theory closed under the rules of $\mathrm{b}^{3}$. In addition, $\mathcal{T}$ is regular (since $\mathrm{Cn} \Gamma[\mathrm{Lt} i]$ is regular) and consistent (since otherwise $A \in \mathcal{T}$ by ECQ). Thus, $\mathcal{T}$ generates a $\mathcal{T}$-interpretation $I_{\mathcal{T}}$ such that, by Lemma 9.5, $T \in I_{\mathcal{T}}(\Gamma)$ (since $T \in I_{\mathcal{T}}(\mathcal{T})$ ) but $T \notin I_{\mathcal{T}}(A)$. So, $\Gamma \not \nvdash_{I_{\mathcal{T}}} A$ by Definition 9.3), whence $\Gamma \nvdash_{\mathrm{Lt} i} A$ by Definition 8.4 and Proposition 9.4. (2) Completeness w.r.t. $\vDash_{\mathrm{Mt} i}$ is immediate by (1) and Proposition 8.5.

If $\Gamma$ is the empty set, let Lt $i$ be the set of all theorems of Lti. Then, Lt $i \nvdash_{\mathrm{Lt} i} A$ and we can proceed similarly as in cases (1) and (2) above. 


\section{Concluding remarks}

We end the paper with some remarks.

1. As pointed out above, Lt4 is equivalent to Łukasiewicz's Ł3 and Lt5 is the logic $\mathrm{G} 3 \mathrm{E}_{\mathrm{t}}^{\leq}$investigated in [15]. In addition, Lt1 is mentioned in p. 65 of [13] (cf. [13] and references therein). The three remaining logics investigated in the paper have not been treated in the literature as far as we know (but cf. the introduction to the paper, where [14] and [19] in the references are briefly commented upon).

2. Given the axiomatizations provided in Definitions 7.1 and 7.2 , it is possible to define all Lt $i$-logics more conspicuously and economically than in Definitions 7.1 and 7.2. For instance, consider Example 10.1 where Lt3 and Lt4 (i.e., Lukasiewicz's Ł3) are defined as negation expansions of the positive fragment of Lewis' S4 (cf. [11]) and contractionless positive intuitionistic logic, respectively.

Example 10.1 Lt3

Axioms: (a1) $A \rightarrow A$; (a2) $(A \wedge B) \rightarrow A /(A \wedge B) \rightarrow B ;($ a3) $[(A \rightarrow$ $B) \wedge(A \rightarrow C)] \rightarrow[A \rightarrow(B \wedge C)] ;(a 4) A \rightarrow(A \vee B) / B \rightarrow(A \vee B)$; $(a 5)[(A \rightarrow C) \wedge(B \rightarrow C)] \rightarrow[(A \vee B) \rightarrow C] ;(a 6)[A \wedge(B \vee C)] \rightarrow$ $[(A \wedge B) \vee(A \wedge C)] ;($ ar $)[A \rightarrow(B \rightarrow C)] \rightarrow[(A \rightarrow B) \rightarrow(A \rightarrow C)]$; (a8) $(A \rightarrow B) \rightarrow[C \rightarrow(A \rightarrow B)] ;(a 9)(A \rightarrow \neg B) \rightarrow(B \rightarrow \neg A)$; (a10) $(\neg A \rightarrow B) \rightarrow(\neg B \rightarrow A) ;($ a11 $)(\neg A \wedge B) \rightarrow(A \rightarrow B) ;$ (a12) $\neg A \rightarrow[A \vee(A \rightarrow B)] ;($ a13 $)(A \vee \neg B) \vee(A \rightarrow B)$

Rules of inference: (MP) $A \rightarrow B$ \& $A \Rightarrow B$; (Adj) $A \& B \Rightarrow A \wedge B$; $(d E C Q) C \vee(A \wedge \neg A) \Rightarrow C \vee B$.

Lt4

Axioms: a1-a6 of Lt3 plus (a7) $(A \rightarrow B)] \rightarrow[(B \rightarrow C) \rightarrow(A \rightarrow C)]$; (a8) $A \rightarrow(B \rightarrow A)$; (a9) $(A \rightarrow \neg B) \rightarrow(B \rightarrow \neg A)$; (a10) $(\neg A \rightarrow B) \rightarrow$ $(\neg B \rightarrow A) ;($ a11 $)[(A \rightarrow B) \wedge A] \rightarrow(\neg A \vee B) ;($ a12 $) \neg(A \rightarrow B) \rightarrow$ $(A \wedge \neg B) ;($ a13 $)(A \vee \neg B) \vee(A \rightarrow B)$.

Rules: $(M P) A \rightarrow B \& A \Rightarrow B ;(d M P) C \vee(A \rightarrow B) \& C \vee A \Rightarrow C \vee B$; $(d E C Q) C \vee(A \wedge \neg A) \Rightarrow C \vee B$.

3. It is possible to endow non-natural implicative logics with a BD-semantics. For instance, in Example 10.2 we have a negation expansion of classical positive propositional logic not included in classical propositional logic.

Example 10.2 Consider, for example, the expansion of MK3 based upon the following table for the conditional (2 is the only designated value)

\begin{tabular}{l|lll}
$\rightarrow$ & 0 & 1 & 2 \\
\hline 0 & 2 & 2 & 2 \\
1 & 2 & 2 & 2 \\
2 & 1 & 0 & 2
\end{tabular}


The logic determined by this expansion can be axiomatized by adding to $b_{1}^{3}$ the following axioms: $A \rightarrow[(B \vee \neg B) \vee \neg(A \rightarrow B)] ; \neg(A \rightarrow B) \rightarrow A$; $[\neg(A \rightarrow B) \wedge B] \rightarrow \neg A$ and $[\neg(A \rightarrow B) \wedge \neg B] \rightarrow \neg A$, but the last axiom is not, of course, a classical tautology.

We note that Baaz' delta operator is definable in this system by using the following formula $A \wedge[(A \rightarrow A) \rightarrow A]$ (we owe this remark to a referee of the JANCL - cf. the paragraph before displaying the structure of the paper in the introduction to it.)

4. The two $\mathrm{Eb}_{1}^{3}$-logics considered in this paper contain classical positive propositional logic but lack the rule Contraposition (if $A \rightarrow B$, then $\neg B \rightarrow \neg A)$.

5. All Lt $i$-logics are paracomplete in the sense that not for any prime, regular and consistent theory $\mathcal{T}$, we have $A \in \mathcal{T}$ or $\neg A \in \mathcal{T}$ for any wff $A$.

Acknowledgements. - This work is supported by the Spanish Ministry of Economy, Industry and Competitiveness under Grants [FFI2014-53919-P; FFI201782878-P]. - We sincerely thank two referees of the JANCL for their comments and suggestions on a previous draft of this paper.

\section{References}

[1] Anderson, A. R., Belnap, N. D. J. (1975). Entailment. The Logic of Relevance and Necessity, vol I. Princeton, NJ: Princeton University Press.

[2] Arieli, O., Avron, A. (1996). Reasoning with logical bilattices. Journal of Logic, Language and Information, 5, 25-63.

[3] Arieli, O., Avron, A. (1998). The value of the four values. Artificial Intelligence, 102, 97-141.

[4] Avron, A. (1991). Natural 3-Valued Logics-Characterization and Proof Theory. Journal of Symbolic Logic, 56(1), 276-294.

[5] Belnap, N. D. Jr. (1977a). A useful four-valued logic. In G. Epstein \& J. M. Dunn (Eds.), Modern Uses of Multiple-Valued Logic (pp. 8-37). D. Reidel Publishing Co., Dordrecht.

[6] Belnap, N. D. J. (1977b). How a computer should think. In G. Ryle (Ed.), Contemporary Aspects of Philosophy (pp. 30-55). Oriel Press Ltd., Stocksfield.

[7] Brady, R. T. (1982). Completeness Proofs for the Systems RM3 and BN4. Logique et Analyse, 25, 9-32. 
[8] Dunn, J. M. (1976). Intuitive semantics for first-degree entailments and "coupled trees." Philosophical Studies, 29, 149-168.

[9] Dunn, J. M. (2000). Partiality and its Dual. Studia Logica, 65, 5-40. http://doi.org/10.1023/A:1026740726955.

[10] González, C. (2012). MaTest. Retrieved from http://ceguel.es/matest (last accessed 04/01/2018).

[11] Hacking, I. (1963). What is Strict Implication? Journal of Symbolic Logic, 28(1), 51-71.

[12] Kleene, S. C. (1962). Introduction to Metamathematics. North Holland. Reprinted Ishi Press, 2009.

[13] Kracht, M. (1998). On Extensions of Intermediate Logics by Strong Negation. Journal of Philosophical Logic, 27(1), 49-73. http://doi.org/10.1023/A:1004222213212.

[14] Omori, H., Sano, K. (2015). Generalizing Functional Completeness in Belnap-Dunn Logic. Studia Logica, 103(5), 883-917. https://doi.org/10.1007/s11225-014-9597-5.

[15] Robles, G., Méndez, J. M. (2014). A paraconsistent 3-valued logic related to Gödel logic G3. Logic Journal of the IGPL, 22(4), 515-538. http://doi.org/10.1093/jigpal/jzt046.

[16] Robles, G., Méndez, J. M. (2019). Belnap-Dunn semantics for natural implicative expansions of Kleene's strong three-valued matrix with two designated values. Journal of Applied Non-Classical Logics, 29(1), 37-63. https://doi.org/10.1080/11663081.2018.1534487.

[17] Robles, G., Salto, F., Méndez, J. M. (2014). Dual Equivalent Two-valued Under-determined and Over-determined Interpretations for Łukasiewicz's 3-valued Logic Ł3. Journal of Philosophical Logic, 43(2/3), 303-332. http://doi.org/10.1007/s10992-012-9264-0.

[18] Routley, R. Meyer, R. K., Plumwood, V., Brady R. T. (1982). Relevant Logics and their Rivals, vol. 1. Atascadero, CA: Ridgeview Publishing Co.

[19] Sano, K., Omori, H. (2014). An expansion of first-order Belnap-Dunn logic. Logic Journal of the IGPL, 22(3), 458-481. https://doi.org/10.1093/jigpal/jzt044.

[20] Tomova, N. (2012). A Lattice of implicative extensions of regular Kleene's logics. Reports on Mathematical Logic, 47, 173-182. http://doi.org/10.4467/20842589RM.12.008.0689. 